Semantics of Prepositional Constructs in Russian:

Tentative Approach

- Adam G. Woyna -

INTERNATIONAL CONFERENCE

ON

C"ÖMPUTATIONAL L L ING U ISTICS

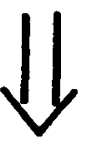

C O L I N G

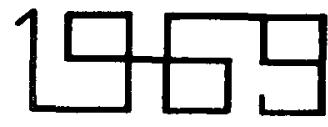

RESEARCH GROUP FOR QUANTITATIVE LINGUISTICS 
SEMANTICS OF PREPOSITIONAL CONSTRUCTS IN RUSSIAN: TENTATTVE APPROACH

Adam G. Woyna

ERIC Clearinghouse for Linguistics

Center for Applied Linguistics, Washington, D. C.

ABSTRACT

The paper describes an experiment designed to investigate the semantic relationships between the three basic components of a prepositional construct: the governor, preposition and the complement. The data was derived from the Soviet Academy of Sciences Grammar and to some extent from the Soviet Academy of Sciences Dictionary. Following the classification provided by the above mentioned grammar, the prepositional constructs were coded according to the grammatical category of their governors and the type of relationship with their complement. The following governor categories were recognized: noun, verb, adjective, adverb, numeral. Next, the governors as well as the complements were coded according to their semantic properties from examples provided by the Grammar. The semantic classification is arbitrary in that it does not attempt to map the universe of a11 Russian word forms in the five governor categories, but only tends to accomodate those complements and governors which occur in the examples and only if their characteristics are relevant. An attempt was made to keep the number of classes at minimum to permit easier recognition of possible patterns. Initial results are presented in tabular form.

Grateful acknowledgement is made to Dr. M. Pacak for many helpful suggestions. 
The primary objective of this paper is to describe an experiment designed to investigate the semantic relationships between the three basis components of a prepositional construct: the governor, preposition and the complement. Because of the preliminary nature of the experiment, only simple data processing equipment, such as the keypunch and the sorter, was used. The implementation of this approach on a larger scale, however, would necessitate the use of more sophisticated hardware.

The described procedure uses Russian prepositions because, while working on this problem, the author was a research staff member of the Russian-English mechanical translation group at IBM's Thomas J. Watson Research Center in Yorktown Heights, New York.

While the described procedure presents a tentative approach, which does not offer a solution to the semantic ambiguities within prepositiona1 constructs in Russian, it does suggest a method for examining each basic component of a given construct in relation to other constructs containing different types of prepositions.

The data used in the model was collected mainly from the Soviet Academy of Sciences Grammar and, to some extent, from the Soviet Academy of Sciences Dictionary. Initially an attempt was also made to compile data from other dictionaries. It was found, however, that the presentation and the classification of the data was not detailed enough for the purposes of this study. Therefore, only some of the 
prepositions not listed as such in the previously named sources were included in the experiment. The next logical step, using the arrangement of the data as shown below, should be the culling out of additiona1 data in the case of Russian, and complete data in the case of other languages, from dictionaries, concordances and random texts. Following various sorting patterns, the results should then be tested through generative processes and checked against concorded 'real 1 ife' examples. Genera1 Purpose

As stated earlier, the purpose of the proposed approach is the establishment of patterns of semantic correlations between:

1. Given Governor and its Preposition $\mathrm{G} \longleftrightarrow \mathrm{P}$ (1eft boundaries)

2. Given Preposition and its Complement $\mathrm{P} \longrightarrow \mathrm{C}$ (right boundaries)

3. Given Governor and its Preposition's Complement

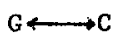

These relationships can be diagrammed as follows:

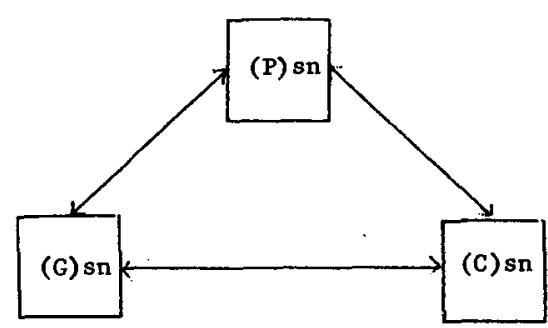

where sn=sematic property of any value. 
If either of the semantic components is found to exclusively govern the combination of the two remaining semantic components then it can be said that

$(G) s n+(C) s n+(P) s n=S x$

where

Sx $=$ valid semantic pattern $=$ sum of semantic properties of

$(G) \operatorname{sn}(C) s n$ and $(P) s n$

Where confirmed, the established semantic patterns can be incorporated into a look-up table as an additional tool for parsing procedures and for testing the validity of class-membership within prepositional phrases. Where not confirmed or where the patterns are not clear, further refinement of the semantic properties of the components in question must be carried out.

\section{Method of Procedure}

Following the classification provided by the Academy of Sciences Grammar (henceforth referred to only as Grammar), prepositional constructs were coded according to the grammatical category of their governors and the type of relationship (henceforth referred to only as TR) with their complement. The following governor categories were recognized: noun, verb, adjective, adverb, numeral.

Next, the governors as well as the complements were coded according to their semantic properties from examples provided by the 
Grammar. This semantic classification was, in a sense, arbitrary in that it did not attempt to map the universe of all Russian words in the five governor categories, but tended to accomodate only those complements and governors contained in the examples, and only if their characteristics seemed relevant. Thus, if the Grammar provided two examples of the usage of the preposition and the semantic properties of respective governors and complements were clearly identical, only one example was selected. The listing of these constructs in the five governor categories is given in Appendix I.

Since, in an initial study of this type, a large number of semantic classes might tend to obscure the existence of possible patterns, an attempt was made to keep the number of these classes at a minimum. As stated earlier, the adoption of this approach in an extensive study of constituents within prepositional constructs would require more elaborate semantic mapping. For the purposes of this study, the total number of semantic classes for nouns was narrowed to 24 , for verbs 9 and 6 for adjectives. (See Appendix II)

The classification of numerals and adverbs as governors was abandoned when it was discovered that, according to the examples provided by the Grammar, their semantic values in no instance effected the selection of a complement of a given semantic category. Thus the 
examples citing the usage of cardinal, ordinal and indeterminate numbers such as "HECKO 6 6KO" showed that these governors may co-occur with a complement of any class. A similiar phenomenon was observed in the behavior of adverbs acting as governors. It is possible that a more detailed study of a large corpus will reveal the existence of definite relationships.

The total number of types of relationships (TR) abstracted from the Grammar was 42 (see Appendix III). Since some of the prepositions, especially of the compound or adverbial type, were not provided by the Grammar with an example of their usage in a given TR, the latter was derived through transformational cross-reference from the Acaderny of Sciences Dictionary, and when that proved impossible, it was supplied by the native speaker of Russian employed by the Project.

In order to fit the data for each TR on a single IBM card (for easier sorting), those TRs which seemed somewhat redundant or insufficiently documented were combined and the tota 1 number of TRs was reduced to 11. Again, while the TRs were translated literally from the Grammar (admitedly, some of the translations seem a little awkward, e. g. 'togetherness'), the reduction of their total number was an arbitrary arrangement aimed at simplifying the overall research procedure. The manner in which the 43 TRs were reduced to 11 is shown in Appendix IV. 
The 11 TRs used in the final analysis are as follows:

$\begin{array}{lcl}\text { 1. Attributive } & 7 . & \text { TR expressing togetherness } \\ \text { 2. Tempora1 } & 8 . & \text { similarity } \\ \text { 3. Spatial } & 9 . & \text { deprivation } \\ \text { 4. Purpose } & 10 . & \text { conformity } \\ \text { 5. Causative } & 11 . & \text { comparison } \\ \text { 6. Objective } & & \\ \text { In addition to the aforementioned, the following data was added }\end{array}$

to every construct:

1. Genera1 relationship

AA purely attributive

oo $\quad * \quad$ objective

MM $\quad * \quad$ modifying

$\mathrm{XX} \quad$ * other

AO attributive with objective shading

OA objective with attributive shading

etc.

2. Relative position to the complement

A PNP precedes the governor

$P \quad$ PNP follows the governor

$\mathrm{X}$ no preference

3. Structura1 restrictions

$\mathrm{x}$ none

I preposition-complement form an idiom in a given configuration

preposition (e.g. COKNATUROC6 (3 HeCkO.26kO AAZ)

-6- 
P governor - preposition form an idiom in a given configuration

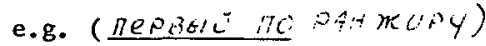

4. Equivalence with other TRs (syntactic difference - semantic equivalence)

1 yes

0 none

5. Other transformational possibilities

1 yes (e.g. (c.2 $5^{A}$ c70.\%0M - ce.2 y cT0.2.4)

0 none

6. Letter codes to facilitate file search

(usually the first three letters of the preposition)

When punched on IBM card the data had the following format:

Columns

3-23

25

27-31

$33-34$

36

38

$40-41$

43

$45-46$

\section{Data}

preposition

case governed by the preposition in a given TR specific relationship with the complement general relationship with the complement

relative position to the complement

governor class

governor sub-class

structura 1 restrictions

complement class 
(cont.)

Colums Data

48

equivalence with other constructs

50

other transformational possibilities

The final step consisted of sorting the cards on individual

columns for detection of patterns.

$-8-$ 
APPENDIX I

\begin{tabular}{llllll} 
Preposition & Noun & Verb & Adjective Adverb & Numeral \\
& as & as & as & as & as \\
& governor & governor & governor & governor & governor \\
\hline
\end{tabular}

To be read as follows:

(grammatical and semantic categories of governor + semantic category

of complement) $=$ Type of Relationship

Bez $\quad(\mathrm{NA}+\mathrm{A})=\mathrm{AT} \quad(\mathrm{VX}+\mathrm{K})=\mathrm{AT}$

$(V X+B)=O B$

Blagodar4*

$(\mathrm{VX}+\mathrm{A})=\mathrm{CA}$

Bliz $\quad(\mathrm{NF}+\mathrm{B})=\mathrm{AT}$

Cerez

$(\mathrm{NM}+\mathrm{Q})=\mathrm{AT} \quad(\mathrm{VX}+\mathrm{B})=\mathrm{OB}$

$(V X+U)=S P$

$(\mathrm{V} 4+\mathrm{E})=\mathrm{SP}$

$(\mathrm{VX}+\mathrm{R})=\mathrm{TE}$

D14

$(\mathrm{NE}+\mathrm{E})=\mathrm{AT} \quad(\mathrm{VX}+\mathrm{C})=\mathrm{OB} \quad(\mathrm{A} 2+\mathrm{A})=\mathrm{CO}$

$(\mathrm{NK}+\mathrm{B})=\mathrm{AT} \quad(\mathrm{VX}+\mathrm{A})=\mathrm{PU} \quad(\mathrm{A} 2+\mathrm{L})=\mathrm{OB}$

Do

$(\mathrm{NC}+\mathrm{K})=\mathrm{AT} \quad(\mathrm{VX}+\mathrm{Y})=\mathrm{AT} \quad(\mathrm{AX}+\mathrm{Y})=\mathrm{AT}$

$(\mathrm{DX}+\mathrm{S})=\mathrm{TE}$

$(\mathrm{NC}+\mathrm{E})=\mathrm{AT} \quad(\mathrm{VX}+\mathrm{A})=\mathrm{OB} \quad(\mathrm{A} 3+\mathrm{Y})=\mathrm{OB}$

$(N P+E)=A T \quad(V X+B)=S P \quad(A 1+E)=S P$

$(\mathrm{NE} \rightarrow \mathrm{B})=\mathrm{AT} \quad(\mathrm{VX}+\mathrm{Q})=\mathrm{TE} \quad(\mathrm{A} 5+\mathrm{Q})=\mathrm{TE}$

$(\mathrm{NL}+\mathrm{B})=\mathrm{SP}$

$(\mathrm{NL}+\mathrm{Q})=\mathrm{TE}$

Iz

$(\mathrm{NC}+\mathrm{A})=\mathrm{AT} \quad(\mathrm{VX}+\mathrm{W})=\mathrm{AT} \quad(\mathrm{AX}+\mathrm{B})=\mathrm{AT} \quad(\mathrm{DX}+\mathrm{A})=\mathrm{SP} \quad(\mathrm{RX}+\mathrm{B})=\mathrm{AT}$

$(\mathrm{NF} \bullet \mathrm{B})=\mathrm{AT} \quad(\mathrm{VX}+\mathrm{V})=\mathrm{CA}$

$(\mathrm{NA}+\mathrm{E})=\mathrm{SP} \quad(\mathrm{VX}+\mathrm{E})=\mathrm{OB}$

$* 4=9$

$-9-$ 
Appendix I (cont.)

(cont.)

$(\mathrm{VX}+\mathrm{G})=\mathrm{OB}$

$(\mathrm{VX}+\mathrm{B})=\mathrm{OB}$

$(\mathrm{V} 1+\mathrm{H})=\mathrm{SP}$

$(\mathrm{VX}+\mathrm{K})=\mathrm{OB}$

$(V 3+B)=S P$

I $z$-pod

$(\mathrm{NF}, \mathrm{D})=\mathrm{A} T$

$(\mathrm{NE}+\mathrm{F})=\mathrm{AT}$

$(\mathrm{NL}+\mathrm{E})=\mathrm{AT}$

Iz-za

$(\mathrm{VX}+\mathrm{A})=\mathrm{SP}$

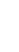

$\mathrm{K}$

$(\mathrm{VX}+\mathrm{B})=\mathrm{CA}$

$(\mathrm{VX}+\mathrm{K})=\mathrm{CA}$

$(\mathrm{NI}+\mathrm{K})=\mathrm{AT}$ $(\mathrm{NI}+\mathrm{B})=\mathrm{AT}$

$(N K+E)=A T$

$(\mathrm{NE}+\mathrm{B})=\mathrm{AT}$

$(\mathrm{VX}+\mathrm{B})=\mathrm{OB}$

$(\mathrm{VX}+\mathrm{A})=\mathrm{OB}$

$(A X+A)=O B$

$(A X+K)=A T \quad(D X+B)=S P$

$(N L+A)=O B$

$(\mathrm{VX}+\mathrm{Q})=\mathrm{TE}$

$(A X+Y)=S P$

$(N P \neg A)=O B$

Krugom

$(N F+B)=A T$

$(V X+B)=S P$

$(A X+B)=S P$

Mejdu*

$(N A+A)=A T$

$(\mathrm{NL}+\mathrm{B})=\mathrm{SP}$

$(\mathrm{VX}+\mathrm{Q})=\mathrm{TE} \quad(\mathrm{AX}+\mathrm{D})=\mathrm{TO}$ $(\mathrm{NK}+\mathrm{A})=\mathrm{TO}$

Mimo

$(\mathrm{VX}+\mathrm{B})=\mathrm{SP}$

$\mathrm{Na}$ acc.

$(\mathrm{NB}+\mathrm{A})=\mathrm{AT}$ $(\mathrm{NL}+\mathrm{A})=\mathrm{AT}$ $(\mathrm{NI}+\mathrm{H})=\mathrm{AT}$

$(\mathrm{NC}+\mathrm{E})=\mathrm{OB}$

$(\mathrm{NL}+\mathrm{A})=\mathrm{OB}$

$(\mathrm{NL}+\mathrm{K})=\mathrm{PU}$

$(\mathrm{NL}+\mathrm{B})=\mathrm{SP}$

$(N N+A)=S P$

$(\mathrm{NM}+\mathrm{H})=\mathrm{SP}$

$(\mathrm{NO}+\mathrm{B})=\mathrm{SP}$

$(\mathrm{VX}+\mathrm{Y})=\mathrm{AT} \quad(\mathrm{AX}+\mathrm{A})=\mathrm{AT}$

$(\mathrm{VX}+\mathrm{F})=\mathrm{AT} \quad(\mathrm{AX}+\mathrm{Y})=\mathrm{AT}$

$(\mathrm{VX}+\mathrm{V})=\mathrm{AT} \quad(\mathrm{AX} \rightarrow \mathrm{B})=\mathrm{OB}$

$(\mathrm{VX}-\mathrm{E})=\mathrm{AT}$

$(\mathrm{VX}+\mathrm{A})=\mathrm{OB}$

$(\mathrm{V} 8+\mathrm{A})=\mathrm{PU}$

$(\mathrm{VX}+\mathrm{B})=\mathrm{SP}$

$(\mathrm{VX}+\mathrm{H})=\mathrm{SP}$

$(\mathrm{VX}+\mathrm{R})=\mathrm{TE}$

$-10-$

$* j=m$ 
Appendix I (cont.)

\begin{tabular}{|c|c|c|c|c|c|}
\hline Na prep. & $\begin{array}{l}(\mathrm{NB}+\mathrm{E})=\mathrm{AT} \\
(\mathrm{NL}+\mathrm{B})=\mathrm{AT} \\
(\mathrm{NL} \bullet \mathrm{Q})=\mathrm{AT} \\
(\mathrm{NL} \bullet \mathrm{E})=\mathrm{OB}\end{array}$ & $\begin{array}{l}(\mathrm{V} 2+\mathrm{E})=\mathrm{AT} \\
(\mathrm{VX}+\mathrm{Y})=\mathrm{AT} \\
(\mathrm{V} 2+\mathrm{F})=\mathrm{OB} \\
(\mathrm{VX}+\mathrm{A})=\mathrm{OB} \\
(\mathrm{VX}+\mathrm{K})=\mathrm{SP} \\
(\mathrm{VX}+\mathrm{F})=\mathrm{SP} \\
(\mathrm{VX}+\mathrm{J})=\mathrm{SP} \\
(\mathrm{VX}+\mathrm{E})=\mathrm{SP} \\
(\mathrm{VX}+\mathrm{Q})=\mathrm{TE}\end{array}$ & $\begin{array}{l}(\mathrm{AX}+\mathrm{Y})=\mathrm{AT} \\
(\mathrm{AX}+\mathrm{E})=\mathrm{SP} \\
(\mathrm{AX}+\mathrm{S})=\mathrm{TE}\end{array}$ & & $(\mathrm{RX}+\mathrm{E})=\mathrm{AT}$ \\
\hline $\mathrm{Nad}$ & $\begin{array}{l}(\mathrm{NL}+\mathrm{B})=\mathrm{AT} \\
(\mathrm{NL}+\mathrm{Y})=\mathrm{OB}\end{array}$ & $\begin{array}{l}(V X+Y)=O B \\
(V X+B)=S P\end{array}$ & & $(D X+B)=S P$ & \\
\hline Naperekor & & $(V X+A)=O B$ & & & \\
\hline Naprotiv & & $(V X+B)=S P$ & & & \\
\hline Nascet* & $(\mathrm{NE}+\mathrm{A})=\mathrm{AT}$ & & & & \\
\hline Navstrecu & & $\begin{array}{l}(V X+L)=S P \\
(V X+B)=S P\end{array}$ & & & \\
\hline Vnutri & & $(V X+B)=S P$ & & & \\
\hline 0 acc. & $(\mathrm{NL}+\mathrm{B})=\mathrm{OB}$ & $(V X+E)=O B$ & & & \\
\hline 0 prep. & $(\mathrm{NY}+\mathrm{A})=\mathrm{AT}$ & $(V X+A)=O B$ & & & \\
\hline Okolo & $(N F+B)=A T$ & $(\mathrm{VX}+\mathrm{B})=\mathrm{SP}$ & $(A X+E)=S P$ & & \\
\hline OT & $\begin{array}{l}(\mathrm{NE}+\mathrm{B})=\mathrm{AT} \\
(\mathrm{NB}+\mathrm{B})=\mathrm{AT} \\
(\mathrm{NK}+\mathrm{Q})=\mathrm{AT} \\
(\mathrm{NE}+\mathrm{A})=\mathrm{AT} \\
(\mathrm{NL}+\mathrm{E})=\mathrm{AT} \\
(\mathrm{NE}+\mathrm{E})=\mathrm{AT}\end{array}$ & $\begin{array}{l}(\mathrm{VX}+\mathrm{K})=\mathrm{CA} \\
(\mathrm{VX}+\mathrm{A})=\mathrm{OB} \\
(\mathrm{VX}+\mathrm{H})=\mathrm{SP}\end{array}$ & $\begin{array}{l}(A X+Y)=C A \\
(A X+A)=D E \\
(A X+B)=S P \\
(A X+Q)=T E\end{array}$ & $(D X+B)=S P$ & $(\mathrm{RX}+\mathrm{B})=\mathrm{AT}$ \\
\hline Otnosite16no & $(\mathrm{NE}+\mathrm{A})=\mathrm{AT}$ & & & & \\
\hline
\end{tabular}

$-11-$

$*_{c}=4$ 
Appendix I (cont.)

\begin{tabular}{|c|c|c|c|c|c|}
\hline Pered & $\begin{array}{l}(\mathrm{NB}+\mathrm{B})=\mathrm{AT} \\
(\mathrm{NL}+\mathrm{B})=\mathrm{AT} \\
(\mathrm{NL}+\mathrm{Q})=\mathrm{AT} \\
(\mathrm{NR}+\mathrm{S})=\mathrm{AT} \\
(\mathrm{NK}+\mathrm{B})=\mathrm{OB} \\
(\mathrm{NL}+\mathrm{A})=\mathrm{OB}\end{array}$ & $\begin{array}{l}(\mathrm{VX}+\mathrm{A})=\mathrm{OB} \\
(\mathrm{V} 2+\mathrm{A})=\mathrm{SP} \\
(\mathrm{VX}+\mathrm{Q})=\mathrm{TE}\end{array}$ & $\begin{array}{l}(\mathrm{AX}+\mathrm{A})=\mathrm{AT} \\
(\mathrm{AX}+\mathrm{B})=\mathrm{OB} \\
(\mathrm{AX}+\mathrm{S})=\mathrm{TE}\end{array}$ & $(\mathrm{DX}+\mathrm{M})=\mathrm{TE}$ & \\
\hline Po acc. & $\begin{array}{l}(\mathrm{NB}+\mathrm{B})=\mathrm{AT} \\
(\mathrm{NA}+\mathrm{A})=\mathrm{AT} \\
(\mathrm{NJ}+\mathrm{Y})=\mathrm{AT}\end{array}$ & $\begin{array}{l}(\mathrm{VX}+\mathrm{E})=\mathrm{SP} \\
(\mathrm{VX}+\mathrm{Y})=\mathrm{AT} \\
(\mathrm{VX}+\mathrm{K})=\mathrm{CA} \\
(\mathrm{VX}+\mathrm{B})=\mathrm{OB} \\
(\mathrm{VX}+\mathrm{H})=\mathrm{SP} \\
(\mathrm{VX}+\mathrm{A})=\mathrm{SP} \\
(\mathrm{VX}+\mathrm{Q})=\mathrm{TE}\end{array}$ & $\begin{array}{l}(A X+E)=S P \\
(A X+E)=A T \\
(A X+Q)=T E\end{array}$ & & \\
\hline Po prep. & & $\begin{array}{l}(\mathrm{VX}+\mathrm{A})=\mathrm{OB} \\
(\mathrm{VX}+\mathrm{L})=\mathrm{TE}\end{array}$ & & $(D X+B)=S P$ & $(R X+E)=A T$ \\
\hline Po casti & & $(\mathrm{VX}+\mathrm{K})=\mathrm{OB}$ & & & \\
\hline Po napravlenih & $\mathrm{k} *$ & $(V X+B)-S P$ & & & \\
\hline Po otnowenih $\mathrm{k}$ & & $(V X+B)=O B$ & & & \\
\hline Po povodu & & $(\mathrm{VX}+\mathrm{A})=\mathrm{CA}$ & & & \\
\hline Po pricine & & $(V X+Y)=C A$ & $(A X+Y)=C A$ & & \\
\hline Po slucah & & $(V X+A)=C A$ & & & \\
\hline Po sravnenih $s$ & & & $(A X+A)=C P$ & & \\
\hline Pod acc. & $\begin{array}{l}(\mathrm{NB}+\mathrm{E})=\mathrm{AT} \\
(\mathrm{NE}+\mathrm{Y})=\mathrm{AT}\end{array}$ & $\begin{array}{l}(\mathrm{VX}+\mathrm{L})=\mathrm{AT} \\
(\mathrm{VX}+\mathrm{A})=\mathrm{OB} \\
(\mathrm{VX}+\mathrm{E})=\mathrm{SP} \\
(\mathrm{V} 2+\mathrm{L})=\mathrm{OB} \\
(\mathrm{VX}+\mathrm{B})=\mathrm{SP} \\
(\mathrm{VX}+\mathrm{R})=\mathrm{TE}\end{array}$ & ' & & \\
\hline & & & & & \\
\hline
\end{tabular}


Appendix I (cont.)

\begin{tabular}{|c|c|c|c|c|}
\hline Pod instr. & $\begin{array}{l}(\mathrm{NF}+\mathrm{F})=\mathrm{AT} \\
(\mathrm{NL}+\mathrm{K})=\mathrm{AT} \\
(\mathrm{NE}+\mathrm{E})=\mathrm{AT} \\
(\mathrm{NL}+\mathrm{E})=\mathrm{SP}\end{array}$ & $\begin{array}{l}(\mathrm{VX}+\mathrm{Y})=\mathrm{AT} \\
(\mathrm{VX}+\mathrm{K})=\mathrm{OB} \\
(\mathrm{V} 6+\mathrm{K})=\mathrm{SP} \\
(\mathrm{VX}+\mathrm{J})=\mathrm{SP} \\
(\mathrm{VX}+\mathrm{E})=\mathrm{SP}\end{array}$ & $(A X+E)=S P$ & $(D X+B)=S P$ \\
\hline Podle & $(\mathrm{NF}+\mathrm{B})=\mathrm{AT}$ & $(V X+B)=S P$ & $(A X+E)=S P$ & \\
\hline Podobno & & $(V X+A)=S I$ & & \\
\hline Poperek & & $(V X+K)=O B$ & & \\
\hline Posle & & $(V X+A)=T E$ & $(A X+Y)=T E$ & \\
\hline Posredstvom & & $(\mathrm{VX}+\mathrm{K})=\mathrm{AT}$ & & \\
\hline Poverx* & $(\mathrm{NF}+\mathrm{B})=\mathrm{AT}$ & $(V X+B)=S P$ & & \\
\hline Pozadi & & $(V X+B)=S P$ & & \\
\hline Prejde & & $(V X+B)=T E$ & & \\
\hline Pri & & $\begin{array}{l}(\mathrm{VX}+\mathrm{K})=\mathrm{AT} \\
(\mathrm{VX}+\mathrm{E})=\mathrm{SP} \\
(\mathrm{VX}+\mathrm{D})=\mathrm{TE}\end{array}$ & $\begin{array}{l}(A X+A)=A T \\
(A X+K)=T E\end{array}$ & \\
\hline Pro & & $\begin{array}{l}(\mathrm{VX}+\mathrm{A})=\mathrm{OB} \\
(\mathrm{VX}+\mathrm{K})=\mathrm{PU}\end{array}$ & & \\
\hline Protiv & $\begin{array}{l}(\mathrm{NB} \bullet \mathrm{K})=\mathrm{AT} \\
(\mathrm{NF}-\mathrm{B})=\mathrm{AT} \\
(\mathrm{NL} \bullet \mathrm{B})=\mathrm{AT}\end{array}$ & $(V X+A)=O B$ & & \\
\hline Putem & & $(V X+K)=A T$ & & \\
\hline R4dom s & & & $(A X+B)=A T$ & \\
\hline Radi & & $(V X+C)=O B$ & & \\
\hline S acc & & $(V X+T)=A T$ & & \\
\hline & \multicolumn{4}{|c|}{-13} \\
\hline
\end{tabular}


Appendix I (cont.)

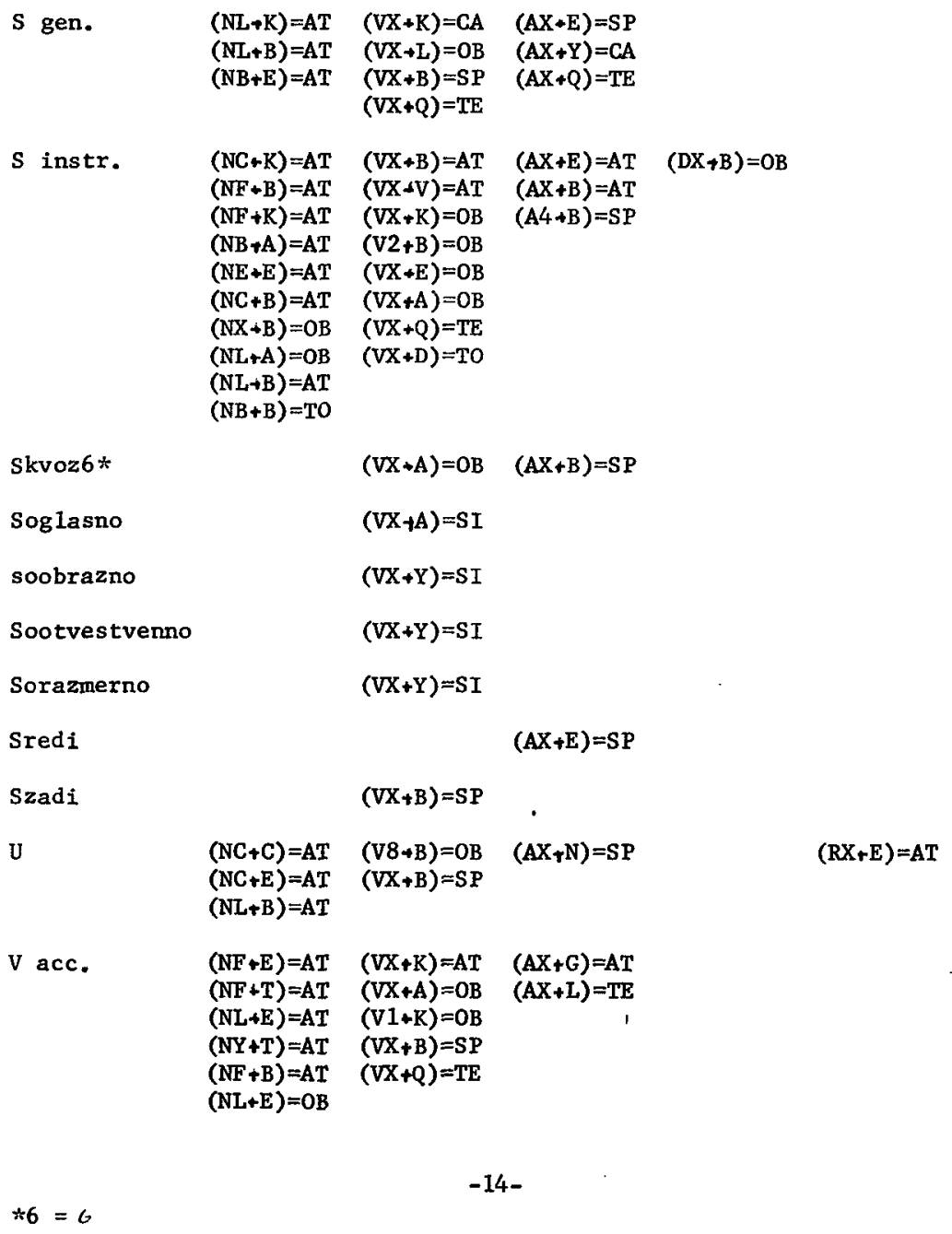




\begin{tabular}{|c|c|c|c|c|c|}
\hline (cont.) & $\begin{array}{l}(\mathrm{NL}+\mathrm{A})=\mathrm{PU} \\
(\mathrm{NL}+\mathrm{B})=\mathrm{SP} \\
(\mathrm{NL}+\mathrm{Q})=\mathrm{TE}\end{array}$ & & & & \\
\hline V prep. & $\begin{array}{l}(N B+E)=A T \\
(N L+B)=A T \\
(N A+B)=A T \\
(N B+B)=O B \\
(N L+B)=O B \\
(N K+K)=O B\end{array}$ & $\begin{array}{l}(\mathrm{VX}+\mathrm{K})=\mathrm{AT} \\
(\mathrm{VX}+\mathrm{E})=\mathrm{AT} \\
(\mathrm{V} 7+\mathrm{K})=\mathrm{OB} \\
(\mathrm{V} 8+\mathrm{K})=\mathrm{OB} \\
(\mathrm{VX}+\mathrm{A})=\mathrm{OB} \\
(\mathrm{VX}+\mathrm{E})=\mathrm{SP} \\
(\mathrm{VX}+\mathrm{U})=\mathrm{SP} \\
(\mathrm{VX}+\mathrm{Q})=\mathrm{TE}\end{array}$ & $\begin{array}{l}(\mathrm{AO}+\mathrm{M})=\mathrm{AT} \\
(\mathrm{A} 3+\mathrm{N})=\mathrm{AT} \\
(\mathrm{AX}+\mathrm{Y})=\mathrm{AT} \\
(\mathrm{AX}+\mathrm{E})=\mathrm{SP}\end{array}$ & $(\mathrm{DX} \bullet \mathrm{B})=\mathrm{SP}$ & $(\mathrm{RX}+\mathrm{E})=\mathrm{AT}$ \\
\hline V dele & & $(\mathrm{VX}+\mathrm{K})=\mathrm{OB}$ & & & \\
\hline V oblasti & & $(V X+K)=0 B$ & & & \\
\hline $\mathrm{v}$ otnowenih $\mathrm{k}$ & & & $(A X+A)=A T$ & & \\
\hline$V$ otnowenii & & $(\mathrm{VX}+\mathrm{K})=\mathrm{OB}$ & & & \\
\hline $\mathrm{V}$.prodoljenie & & $(\mathrm{VX}+\mathrm{Q})=\mathrm{TE}$ & & & \\
\hline V qe14x* & & $(\mathrm{VX}+\mathrm{K})=\mathrm{PU}$ & & & \\
\hline v silu & & $(V X+Y)=C A$ & & & \\
\hline $\mathrm{V}$ tecenie & & $(\mathrm{VX}+\mathrm{Q})=\mathrm{TE}$ & & & \\
\hline Vblizi & $(N F+B)=A T$ & $(\mathrm{VX}+\mathrm{B})=\mathrm{SP}$ & & & \\
\hline Vdo16 & $(\mathrm{NF}+\mathrm{B})=\mathrm{AT}$ & $(V X+B)=S P$ & & & \\
\hline Vmesto & $(\mathrm{NA}+\mathrm{A})=\mathrm{OB}$ & & & & \\
\hline Vnutr6 & & $(\mathrm{VX}+\mathrm{B})=\mathrm{SP}$ & & & \\
\hline Vokrug & $(\mathrm{NF}+\mathrm{B})=\mathrm{AT}$ & $(\mathrm{VX}+\mathrm{B})=\mathrm{SP}$ & & & \\
\hline Vopreki & & $(V X+A)=O B$ & & & \\
\hline$* q=4$ & & -1 & & & \\
\hline
\end{tabular}


Appendix I (cont.)

\begin{tabular}{|c|c|c|c|}
\hline Vozle & $(\mathrm{NF}+\mathrm{B})=\mathrm{AT}$ & $(V X+B)=S P$ & $(\mathrm{AX}+\mathrm{E})=\mathrm{SP}$ \\
\hline Vperedi & & $(V X+B)=S P$ & \\
\hline Vsled & & $(V X+B)=S P$ & \\
\hline Vsledstvie & & $(V X+Y)=C A$ & \\
\hline Vvidu & & $(V X+Y)=C A$ & \\
\hline Vzamen & $(N A+A)=O B$ & & \\
\hline $\mathrm{Za} \mathrm{acc.}$ & $\begin{array}{l}(\mathrm{NL}+\mathrm{K})=\mathrm{CA} \\
(\mathrm{NL}+\mathrm{A})=\mathrm{OB} \\
(\mathrm{NL}+\mathrm{R})=\mathrm{AT} \\
(\mathrm{NL}+\mathrm{J})=\mathrm{SP} \\
(\mathrm{NL}+\mathrm{R})=\mathrm{TE}\end{array}$ & $\begin{array}{l}(\mathrm{VX}+\mathrm{A})=\mathrm{OB} \\
(\mathrm{VX}+\mathrm{B})=\mathrm{OB} \\
(\mathrm{VX}+\mathrm{Y})=\mathrm{OB} \\
(\mathrm{VX}+\mathrm{B})=\mathrm{SP} \\
(\mathrm{VX}+\mathrm{Q})=\mathrm{TE}\end{array}$ & $\begin{array}{l}(A X+A)=A T \\
(A X+Q)=T E\end{array}$ \\
\hline $\mathrm{Za}$ instr. & $\begin{array}{l}(\mathrm{NB}+\mathrm{B})=\mathrm{AT} \\
(\mathrm{NL}+\mathrm{K})=\mathrm{AT} \\
(\mathrm{NL}+\mathrm{E})=\mathrm{AT} \\
(\mathrm{NL}+\mathrm{A})=\mathrm{OB}\end{array}$ & $\begin{array}{l}(\mathrm{VX}+\mathrm{B})=\mathrm{AT} \\
(\mathrm{V} 2+\mathrm{B})=\mathrm{OB} \\
(\mathrm{V} 5+\mathrm{B})=\mathrm{SP} \\
(\mathrm{VX}+\mathrm{A})=\mathrm{OB} \\
(\mathrm{VX}+\mathrm{S})=\mathrm{TE}\end{array}$ & $(A X+B)=S P$ \\
\hline
\end{tabular}

$(R X+E)=A T$ 
APPENDIX II

Nouns

\begin{tabular}{cc} 
Any noun & A \\
Concrete & B \\
animate & $\mathrm{C}$ \\
personal & $\mathrm{D}$ \\
inanimate & $\mathrm{E}$ \\
objects & $\mathrm{F}$ \\
mass & $\mathrm{G}$ \\
location & $\mathrm{H}$ \\
$\quad$ geographical (town, etc.) & $\mathrm{I}$ \\
& $\mathrm{J}$ \\
Abstract etc.) & $\mathrm{K}$ \\
Deverba1 & $\mathrm{L}$ \\
transitive & $\mathrm{M}$ \\
intransitive & $\mathrm{N}$ \\
capable of taking prefix NA & $\mathrm{O}$ \\
Adjectival & $\mathrm{P}$ \\
Nouns designating TIME & $\mathrm{Q}$ \\
days, year, etc. & $\mathrm{R}$ \\
events (birth, death, dinner, etc.) & $\mathrm{S}$ \\
Measure & $\mathrm{T}$ \\
preceded by a numeral & $\mathrm{U}$ \\
Inner state, emotion & $\mathrm{V}$ \\
Phenomena of nature (rain, frost, etc.) & $\mathrm{W}$ \\
Any inanimate, abstract or concrete noun & $\mathrm{Y}$ \\
\hline
\end{tabular}

$-17-$ 


\section{Appendix II (cont.)}

Verbs

Unspecified $\quad X$

Motion 1

Concrete action (chop, hit, tear, grind, etc.) 2

Motion in the broad sense of the term (swing, jump, bring, etc.)

Motion, intransitive and concrete action 4

Concrete state (sit, hang, stand, lie) 5

Concrete state and motion 6

$\begin{array}{ll}\text { Verb phrase } & 7\end{array}$

Inner state, feeling, inte1lectual activity (grieve,
read, etc.)

Adjectives

Unspecified

Concrete (capable of being perceived and ummistakeably ascertained visually, e.g. tall, wide, white, etc.)

Abstract (denoting state, density, mood, age, temperature, sequence, sensed olfactorily or aurally, etc.) 2

Qualitative (good, bad, difficult, strong, etc.) 3

$-18-$ 
Appendix II (cont.)

Adjectives (cont.)

Denoting distance or location

Inner quality possessed only by humans or animals

$-19-$ 
APPENDIX III

Types of TRs abstracted in the initial stage of the study:
1. Spatia1
19. Separation attributive
2. Tempora 1
20. Resu1t-cause
3. Causative
21. Temporal attributive
4. Purpose
22. Substitution
5. Manner of action
23. Restrictive attributive
6. Objective
24. Property attributive
7. General attributive
25. Objective-restrictive
8. Similarity
26. Restrictive in time
9. Property
27. Similarity attributive
10. Togetherness
28. Designation attributive
11. Possession
29. Characteristic attributive
12. Condition
30. Container and contained attr.
13. Adverbia1 attributive
31. Deprivation
14. Quantitative attributive
32. Removal (ablation)
15. Designation
33. Conformity
16. Attributive of absence
34. Separation
17. Origin
35. Non-conformity
18. Spatia1 attributive
36. Comparative

$-20-$ 
Appendix III (cont.)

37. class belonging

38. Detached object

39. Substitutability

40. Advocacy or defence

41. Transgressive

42. Distributive

The TRs are listed in order of their description in the Grammar. 


\section{APPENDIX IV}

The 42 TRs were distributed into 11 major TRs as follows:

1. Spatial

2. Tempora1

Restrictive in time

3. Causative

4. Purpose

5. Objective

Result-cause

Advocacy or defence

Transgressive

Distributive

Detached object

Substitutability

Substitution

objective-restrictive

6. Similarity

7. Togetherness

8. Deprivation

Remova 1

9. Conformity

Non-conformity

10. Comparative
11. Attributive

Manner of action

General attributive

Possession

Condition

Adverbial attributive

Quantitative attributive

Designation

Attributive of absence

Origin

Spatial attributive

Separation attributive

Temporal attributive

Restrictive attributive

Property attributive

Similarity attributive

Designation attributive

Characteristic attributive

Container and contained

attributive

Separation

Restrictive

class belonging 